commend ether or camphor and the salts of caffeine. Among the latter I desire to express a caution as to the use of iodiosalicylate, because I have observed that marked relapse is apt to follow the use of that remedy, whereas the sodiobenzoate and the citrate appear to be exempt from that drawback. It is a well-known fact that digitalis fails to relieve much more frequently in cases of angina pectoris vera than in many other diseases of the heart. This applies as much to the uncomplicated forms caused by the sclerosis of the coronary vessels as to those which are combined with aortic valvular lesions or aortitis. We must not, however, undervalue its usefulness to such an extent as has been done latterly in different directions, for, though the pains be little or not at all influenced by its use, we often succeed in obtaining a beneficial effect from this important drug in cases of heart disease. Still less must we rely upon strophanthus which too often is ineffectual; and still less can we trust to inferior remedies such as convallaria majalis, adonis, and such like remedies which need scarcely be mentioned.

In such cases as we are now considering physical therapy proves its value in a most satisfactory way, and the tonic effect of the balneological and gymnastic treatment as described by my late brother and myself becomes most evident. I abstain from recurring to the details of our system and only lay stress upon the importance of much caution in the application of the baths as well as the therapeutic exercises. I would especially express a warning against hurrying on to strong effervescent baths and especially to the strongest form of the effervescent running baths. Besides, more harm than benefit would be derived from baths either too cool or too warm. Very rarely are we allowed to go below $85^{\circ}$ or to rise above $93^{\circ} \mathrm{F}$., nay, even from a temperature of $95^{\circ} \mathrm{I}$ have now and then observed unfavourable effects. Moreover a long immersion is not advisable. I mostly limit it to the space of 15 minutes and rarely extend it to 20 minutes. Similar precautionary measures are to be observed with regard to exercises. The resistance must not be very strong, the pauses between the exercises must not be too short, and the patient must never be suffered to exert himself till fatigued. A sufficient interval of rest after the exercises is under all circumstances important.

In a series of articles I have shown in past years the results of the balneological and gymnastic treatment especially in cases in which rest as well as pharmaceutic remedies proved useless. Recently their efficacy has been attested by many authorities. In response to inquiries lateiy addressed to me I should be glad to state that the treatment by baths and exercises has proved to be of signal success even in cases in which the nitrites, and principally nitro-glycerine, had more or less failed. I would especially refer to a treatise by Rives. ${ }^{3}$ Rives had the opportunity of observing at Nauheim a patient who was under my treatment and had been sent to me by Sir T. Lauder Brunton. He came from Karachi, was aged 53 years, and was subject to so many stenocardiac fits that in the first few days of his stay at Nauheim he was obliged to have recourse to as many as 20 tabloids of nitro-glycerine a day. A treatment of 13 weeks caused all symptoms not only to disappear but resulted in a general state of well-being. It deserves to be remarked that this patient continued to enjoy good health until great mental strain induced by the ravages of the plague in his town subjected him again to slight attacks. A renewed treatment in Nauheim of seven weeks in the spring and of five weeks in autumn again had the effect of removing his troubles so that Sir T. I sauder Brunton, on the return of the patient, was able to certify a perfectly normal condition of the heart. I must not omit to mention that with such patients it is necessary to adhere to the same precautionary measures as to diet, exercise in fresh air, dress, \&c., as are to be observed with other patients with heart diseases, such as failure of compensation in consequence of valvular lesions, and in myocarditis, \&c.

It must, however, be clearly stated that not all cases without exception are suitable for balneological and gymnastic treatment. When arterio-sclerosis has made such progress that the danger of embolism or apoplexy may be apprehended, or where there is reason to believe that there already exists even a small aneurysm of the wall of the heart or the aorta which might favour rupture, it is well known that every increase of the blood-presspre, which is a natural consequence of the above treatment, might lead to

3 New York Medical Journal, 1896. twe fatal consequences. Rigidity of the vessels or early stages of arterio-sclerosis may be treated by these methods and usually yield satisfactory results, but I must lay stress again upon the fact that advanced sclerosis is a contraindication for this treatment. As regards the latter state I have raised a note of warning years ago, ${ }^{4}$ and my later experience has only strengthened my view on the subject. In such cases strict enforcement of rest is certainly preferable.

Looking, in conclusion, once more at the actual state of our therapeutic experience we are brought to the conclusion that the results which we obtain by the application of the above-mentioned more prominent medicaments is to be explained by the lowering of the pressure of the blood and the moderation of the heart's action consequent upon it. Differing therefrom are the causes which ensure success in the balneological and gymnastic treatment because it is obtained by the exercise of a tonic influence. By strengthening the heart's muscle, as well as by acting on the cardiac nerves, the distressing symptoms of the angina pectoris are either removed or reduced ; in other worde, ex juvantibus, it is shown that the Parry-Stokes theory is based on a real and solid foundation.

Bad-Nauheim.

\section{SOME REMARKS UPON THE TREATMENT OF HEART DISEASE, WITH SPECIAL REFERENCE TO THE "HILL HEART."}

BY H. J. CAMPBELL, M.D., F.R.C.P. LOND.,

SEXIOR PHYSICIAN TO THE BRADFORD ROYAL INFIRMARY AND LECTURER ON FORENSIC MEDICINE IN THE YORKSHIRE COLLEGE, IEEDS.

IN the treatment of most forms of heart disease the difficult problem of how to relieve an organ which has such constant demands made upon it, and which is obliged to rest and recuperate in the brief intervals allowed between its active contractions, is met in one of two ways, the one being the stimulation of the heart, so that by working harder and more efficiently, it may compensate for its own defectiveness, and the other the lessening of the work that the organ has to perform, so that the balance of demand and supply of force may be restored and maintained. In most cases of valvular disease with failing compensation, we of course adopt the two methods of excitation and compensation both at once, combining the exhibition of cardiac stimulants, such as digitalis, strophanthus, convalaria, sparteine. or caffeine, with the diminishing of cardiac work by ordering rest in bed, and the lowering of blood-pressure by the judicious use of purgatives, diuretics, \&c.

In the special methods adopted at Nauheim, the same combination of stimulation with alternate lessening of work, explains the marked success of this form of treatment in suitable cases. The effect of the Sprüdel water at a temperature of $95^{\circ}$ and charged with carbonic acid, is to cause the peripheral vessels to contract and the bloodpressure consequently to rise, as evidenced by the slowing of the pulse. The result is that the heart has more work to do and therefore has its muscle stimulated to more forcible and complete contraction, with the consequent more thorough emptying of its cavities. After leaving the bath the peripheral vessels dilate, the blood.pressure is lowered, and the resulting diminution of the work the heart has to perform, enables the muscle to maintain its increased state of efficiency. Exactly the same sequence of events occurs with the resisted movements, for here again the first effect is to cause a rise of blood-pressure, shown by the slowing of the pulse, while the second effect of dilatation of the vessels of the muscle lowers blood-pressure and therefore lessens the strain upon the labouring heart. In this respect it is interesting to note that Dr. Schott has demonstrated by the use of the Roentgen rays that the more complete emptying of the heart and the consequent reduction of dilatation under this treatment, is an actual fact and is

4 The Lancet, May 23rd, p. 1143, and May 30th, 1891, p. 1199. 1 A paper read before the Yorkshire Branch of the British Medical Association on March 28th, 1900, at Huddersfield. 
not to be explained by better filling of the lungs and consequent diminution of the area of cardiac dulness. The good results of Oertel's method of gradnated walking exercises by slow movements, first on the flat and then up inclined planes, are capable of a similar explanation.

All these methods, however, to be successful must have a heart to work upon which has muscle substance capable of either exerting more force under increased stimulation, of increasing its power of contraction as the result of its hypertrophy if the conditions are favourable, or at least of recovering itself if nutrition is abundant, and partial rest gives it time for recuperation. The cases which, from the point of view of treatment, present the most difficulty are those in which, from whatever cause, the muscle substance is degenerated to such an extent that it is unable to react to the means employed for its relief. In such cases, whether the condition is the last stage of disease of the aortic valves, of failure of the right ventricle in old emphysema and bronchitis, or of the left ventricle in granular kidney, all that can be done is to acknowledge that the heart is no longer able to maintain an efficient circulation of the blood in the adverse circumstances in which it is placed, and to endeavour permanently to lessen the work it has to dothat is to say, to alter as far as possible the relationship between the general system and the heart in such a way that the patient may, for a longer or shorter period, enjoy an impaired but still tolerable degree of comfort and health.

There is another class of case, however, and one which is especially common in hilly districts, where there is also no valvular disease, but where the heart muscle is degenerated to an extent which, though not sufficient to incapacitate the patient from following his ordinary avocations or, as a rule, to produce any backward pressure effects, yet, when any sudden strain is thrown upon it, or any interference with its free movements is encountered by it, is apt to give rise to alarming and dangerous attaoks. These may only cause distress, dyspncea, or anginal pain, or they may induce syncope, or even lead to seizures of an epileptiform or apoplectiform character, and may, and not infrequently do, terminate in almost sudden deatb. The following brief extracts from my notes will serve to point out the main features met with in these cases.

CASE 1. - The patient was a bachelor, aged about 70 years, who had always enjoyed good health, but for the last few years had been unable to walk upstairs so well as formerly and had often had to stop and rest on going up hill. His appetite was good and sleep was not disturbed. During the week before his medical attendant asked me to see him he had had repeated attacks of acute cardiac distress with faintness and a feeling of impending dissolution. He had only been under treatment since these attacks commenced. There was no valvular lesion; the heart beat was fairly forcible and the pulse was regular and not of high tension. All the other organs were healthy. There was no albumin in the arine. During the previous week the patient had suffered from a great deal of flatulence. For two days after seeing the patient there was no attack, then one came on which proved immediately fatal.

CASE 2.-The patient was a man, aged 58 years, who was first seen in May, 1896, and who for several years had had flatulent dyspepsia. On two occasions he had had severe fainting attacks followed by a feeling of great exhaustion necessitating rest in bed and treatment for some weeks. The bowels were confined. The patient looked older than his years and had an anxious expression. He was fairly well nourished, though he stated that lately he had been eating very little in the hope of diminishing the flatulence. On going up hill he often had to stop, not from dyspncea, but from a feeling of oppression on the chest associated with what he described as a "beating" in the stomach. The heart was considerably enlarged, the apex beat was feeble and somewhat diffused, and the pulse was of low tension and very irregular; no bruits were to be heard and all the other organs were healthy, except the stomach, which was dilated. On a light but nourishing diet and general tonic treatment the patient improved and two months later was put upon sparteine sulphate and nitro-glycerine. The next time I heard about him was nearly two years later when, after a long period of comparative comfort, during which he had not required treatment, he had become careless as to his food and mode of life with the result that all his old symptoms returned. On this occasion it was decided, since the condition was of much shorter duration than on the former one, at once to make use of cardiac tonics and he was given a pill of sparteine sul. phate, digitelis, and extract of hyoscyamus once a day. Creasote in mixture was ordered to be taken immediately before meals and he was again put upon a strict diet and directed to move about slowly. 18 months later when seeing another member of the family I casually saw the patient who looked well and said that as long as he was careful he got on all right, a statement that his medical adviser confirmed, bot that any small indiscretion upset bim.

CASE 3.-For purposes of comparison I may briefly mention the following case. The patient was a man, aged 25 years. Until recently he had been accustomed to be in constant training and to spend most of his time in athletic sports; lately, however, he had given up athletics and had very little exercise. In the course of the previous two weeks be had had three syncopal attacks. He was a healthy-looking, welldeveloped, well-nourished man. All organs were normal except the heart which was enlarged, with a feeble diffused impulse, a prolonged first and a somewhat accentuated second sound. The pulse was 75 and was feeble. In this case the condition was evidently one of a "soft" heart-that is to say, of a heart in which the muscle, which during training had become hypertrophied to meet the increased strain pnt upon it, had when the strain was relaxed become flabby. I have not heard from this patient's medical man what the further history of the case was, bat I have no doubt that the condition proved to be a purely temporary one.

CASE 4.-In the last case that I propose to mention the patient was a man, about 65 years of age, who had had a similar attack to the one for which I first saw him, some months before. He was a stout, florid man, had always been temperate, and had had remarkably good health all his life. One night be went to bed in his usual health, but early on the following morning he had an attack which was of a convulsive nature but of short duration. When his medical attendant saw him shortly after the attack he had a bounding pulse and was only semi-conscions, with suffused head and face. His medical attendant performed venesection to ten ounces, after which he improved rapidly. At 2 P.M. on the same day, when we saw him together, there were no localising symptoms, he had completely recovered consciousness, and except for headache did not feel as though there were much the matter with him. The treatment consisted in dieting and the administration of nitroglycerine, under which he soon got about again, but almost every week he had a slight attack which his medical adviser said was not unlike minor epilepsy, but he, however, never lost consciousness. These attacks were specially apt to come on after any excitement or after a full meal. The heart was enlarged and the first sound was thick, but there was no valvular disease. The patient continued in much the same condition until December last, when he had a rather more severe attack, though still without loss of consciousness. The pulse was lower in tension but otherwise the condition was unchanged. Shortly afterwards he had a similar attack which proved fatal.

I have introduced the third case because it explains, I think, why in hilly districts one so frequently meets with the condition illustrated by the other three cases. The history of these cases, I take it, is as follows. A healthy man who is constantly having to go up and down hills, working hard, eating probably somewhat largely, the food, moreover, not always being very easy of digestion, develops cardiac hypertrophy and slight gastric dilatation. As time goes on he probably becomes wealthier and hence most likely takes less exercise, whilst at the same time his food is liable to be richer, although in quantity not materially diminished. If at the same time alcohol be at all freely indulged in, all the circumstances are present which tend to produce dilatation of the stomach and degeneration of the heart walls, the hypertrophied muscle becoming soft and fatty changes occurring in the cells The stomach, too, which has bravely battled for years with the somewhat large supplies which it has been made to bold, now begins to lose tone and stretch. At the same time the peptic and especially the oxyntic cells become less active and fermentative changes are apt to occur in the food owing to its lying too long in the atonic organ, with the result that gases are evolved which still further tend to increase the distension. There is probably, too, less room for the stomach to expand downwards than was previously the case, and it therefore when distended pushes up against the heart, which, degenerated as it is, can but ill cope with any more difficulties than it already constantly has to 
surmount. The whole result is that the heart's action becomes tumultuous, irregular, or imperfect, according to the degree of degeneration which is present in its walls, and the patient experiences a feeling of distress which may proceed no farther or which may lead to so-called lipocardiac asthma, or syncope, or to epileptiform or apoplectiform attacks, any one of which may be more or less suddenly fatal. In cases where the heart muscle, though degenerating, is still able, for at any rate a short time, to respond to the increased excitation, the beats become very forcible and laboured, whilst the intravascular tension is raised and the return of the blood to the heart is interfered with. Under such circumstances there may be increased intracranial pressure, and epileptiform or even apoplectiform attacks with more or less profound unconsciousness may occur. In the latter case the breathing may become stertorous and the whole appearance may be strongly suggestive of cerebral hamorrhage, but if the patient survives a few hours will generally suffice to show the true character of the seizure, for as a rule the attack is of but short duration and there are either no localising symptoms or if there be any muscular weakness it very soon passes off.

As regards treatment, if the disease be in an early stage cure may probably be effected by the absorption of fat and the hypertrophy of the muscle cells, but if the condition be more advanced the treatment presents much difficulty, for whilst almost every case needs its own special line of treatment it is frequently by no means easy to determine what will be beneficial in any individual case. The first requirement always seems to me to be, that the stomach should be treated by strict dieting as well as by gastric antiseptics, such as sulpho-carbolate of soda, creasote in mixture, or salicylate of bismuth, or if necessary by lavage. Further, it should be insisted on that the meals should be taken practically dry, the necessary quantity of fluid being drunk when the stomach is empty. In addition, some saline aperient such as Hunyadi Janos or Apenta water should be taken with a glass of hot water before breakfast. Later artificial digestants, mineral acids, gentian, strychnine, capsicum, \&c., are of service, but it always seems to me that the first need is to clear the stomach and to attempt by preventing distension to reduce its size. As regards the heart itself, the most important thing is to diminish its work, and this may often be accomplished by making the patient rest in bed with a restricted diet, and by the regular exhibition of trinitrin or in rare cases erythrol tetranitrate. The milder cases do not, I think, need to be kept in bed if they can be trusted to move about slowly and to obey directions as to diet. Where the attacks are epileptiform or apoplectiform in character a more marked lowering of blood-pressure by diuretics and free purgation by salines and even sometimes by venesection is advisable. Alcohol, except for sudden emergencies, is, I think, better withheld, or if it be allowed it should only be taken in small quantities with meals. As regards the direct stimulation of the heart I have had, I think, the best results with sparteine sulphate combined with citrate of caffeine. In cases where the heart is rapidly failing beneficial results may sometimes be induced by administering full doses of strophanthus every hour for from 12 to 24 hours and by the inhalation of oxygen over the same period; but unfortunately in most of these cases where once the left ventricle has dilated to any considerable extent it is a sign that the unequal contest will soon be given up for good. When the seizure is over, however, the most important part of the systematic treatment must undoubtedly be directed to the stomach if future attacks are to be prevented. In most, or at any rate in a large number of cases, when they come under observation the stomach is found to be markedly dilated, and without question the onset of an attack, whether simply of distress, of dyspncea, or of a more serious nature is in the large majority of cases associated with flatulent distension of that organ.

"Hill heart," as this condition may be named, differs from fatty degeneration of the heart as generally described in that there is in the "hill heart" antecedent hypertrophy and consequently a greater liability to the apoplectiform and epileptiform kind of attack, both being associated with a temporary increase in the blood-pressure. The resulting high tension is thus due to a conservative constriction of the peripheral arteries and a resulting more forcible action of the heart when its movements are interfered with by a distended stomach or other cause. This, of course, to a considerable extent modifies the treatment and nocessitates a variation in the methods to be employed according to the stage to which the disease has advanced.

With regard to the reflexly excited tumultuous movements it must be remembered that Gaskell, Morison, and others have shown that, contrary to what used to be taught, the whole of the heart substance is abundantly supplied with nerves. Further, as Morison has suggested, it is not improbable that simple hypertrophy with consequent stretching of the intermuscular nerves may, when only slight extra work is thrown upon the heart, cause slight increase of the tension on the nerves sufficient in itself to cause excitation of them, and so the origination of irregular contractions of the muscle, and thus the production of anginal pain.

I have only attempted to deal in some detail with fatty degeneration of the heart, with special reference to degeneration following hypertrophy but without valvular lesion. With such difficult subjects as the treatment of mitral stenosis, of uncomplicated aortic disease, of the sleeplessness of both aortic and mitral disease, and of the vomiting of mitral and tricuspid disease, $I$ have made no attempt to contend, for each one would need a separate paper of its own if justice were to be done to it. Nor have I discussed the value of opium, of jodide, of arsenic, and of mercury in certain heart states, the heart conditions associated with septic disease, the toxic effects of alcohol and tobacco, sypbilis, or the fatty degenerations resulting from too large or too long-continued doses of such drugs as phosphorus and arsenic. Finally, if I have perhaps insisted too much upon the danger of overloading the stomach when the heart is diseased I have only copied Shakespeare, who truly realised the importance of the matter when he made Macbeth say to the doctor who was treating Lady Macbeth :-

Cure ber of that

Canst thou not minister to a mind diseased,

Pluck from the memory a rooted sorrow,

Raze out the written troubles of the brain,

And with some sweet oblivious antidote

Cleanse the stuff'd bosom of that perilous stuff

Bradford. Which weighs upon the heart?

\section{TRACHEOTOMY WITH ANTITOXIN IN LARYNGEAL DIPHTHERIA.}

BY W. BLAIR BELL, M.B. Loxd., M.R.C.S. ENG.

Ix spite of all the personal evidence and in spite of statistics there is still a lurking suspicion in the minds of some medical men and an absolute assuredness on the part of the laity who are of the anti-vivisection persuasion that the antitoxin serum has not fulfilled expectations. Those medical men who are still in doubt must have used it injudiciously - that is, too late or in too small doses, or only in the severest cases, or possibly not at all. Those of the laity who are waging a wordy war against its efficacy cannot have had the opportunity of being grateful to it for its curative action on themselves or those near and dear to them. I have used it continuously in all cases for the last four years and in my limited experience in my own practice I can say that I have not lost a single case directly from the disease. One patient died suddenly from heart failure associated with pneumonia nine weeks after tracheotomy had been performed, but beyond the cardiac weakness following the diphtheria one could hardly attribute the result to that disease. I had also to be personally thankful to it during a recent attack of diphtheria. It is not my intention to dilate on the use of the antitoxin beyond stating that the ordinary dose of 1500 units is quite inadequate for severe cases; and $I$ now never give less than 3000 units for an initial dose, and do not hesitate at 8000 units for any patient over 12 years of age.

The following are notes of three consecutive cases of severe laryngeal diphtheria in which I performed tracheotomy last year. They were the only cases which required the operation, for others which I had during that period yielded to the antitoxin alone. I am convinced that the success attending the operations was due to the antitoxin given. A few years ago they would all probably have proved fatal. It would seem that the antitoxin loosens the existing membrane and prevents fresh membrane from forming, and 